\title{
Stratospheric Balloons for Planetary Science and the Balloon Observation Platform for Planetary Science (BOPPS) Mission Summary
}

\author{
Tibor Kremic \\ NASA Glenn Research Center \\ 21000 Brookpark Road \\ Cleveland, OH 44135 \\ 216-433-5356 \\ Tibor.Kremic@nasa.gov \\ Eliot F. Young \\ SW Research Institute \\ 1050 Walnut Street \\ Boulder, CO 80302 \\ 720-432-2333 \\ efy@boulder.swri.edu
}

\author{
Andrew F. Cheng \\ JHU Applied Physics Lab \\ 11100 Johns Hopkins Road \\ Laurel, MD 20723 \\ (240) 228-5415 \\ Andy.Cheng@jhuapl.edu \\ Rafat R. Ansari \\ NASA Glenn Research Center \\ 21000 Brookpark Road \\ Cleveland, OH 44135 \\ 216-433-5008 \\ Rafat.R.Ansari@nasa.gov \\ Rob R. Landis \\ NASA Wallops Flight Facility \\ 34200 Fulton Street \\ Wallops, VA 23337 \\ 757-824-1641 \\ Rob.R.Landis@nasa.gov
}

\author{
Karl Hibbitts \\ JHU Applied Physics Lab \\ 11100 Johns Hopkins Road \\ Laurel, MD 20723 \\ 240-228-2834 \\ Karl.Hibbitts@jhuapl.edu \\ Matthew D. Dolloff \\ NASA Glenn Research Center \\ 21000 Brookpark Road \\ Cleveland, OH 44135 \\ 216.433-6178 \\ matthew.d.dolloff@nasa.gov
}

\begin{abstract}
NASA and the planetary science community have been exploring the potential contributions of stratospheric balloons to decadal class planetary science. Previous studies of the $\sim 200$ questions raised in the Decadal Survey have identified about 45 topics that are potentially suitable for addressing by stratospheric balloon platforms. A stratospheric balloon mission was flown in the fall of 2014 called BOPPS, Balloon Observation Platform for Planetary Science. This mission observed a number of planetary targets including two Oort cloud comets. The optical system and instrumentation payload was able to provide unique measurements of the intended targets and increase our understanding of these primitive bodies and their implications for us here on Earth. This paper will discuss the mission, instrumentation and initial results and how these may contribute to the broader planetary science objectives of NASA and the scientific community.
\end{abstract}

This paper will also identify how the instrument platform on BOPPS may be able to contribute to future balloon-based science. Finally the paper will address potential future enhancements and the expected science impacts should those enhancements be implemented.

\section{TABLE OF ConTents}

1. INTRODUCTION

2. Summary OF Potential Contributions of Balloon Borne Payloads to Planetary SCIENCE. .2

3. CONTRIBUTIONS OF THE BRRISON AND BOPPS DEVELOPMENTS AND FLIGHTS 3

4. Potential Future SYSTEM AND ENHANCEMENTS To Enable More Planetary Science 7

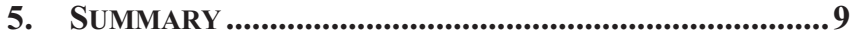

REFERENCES.................................................................99

BIOGRAPHIES...............................................................99

\section{INTRODUCTION}

NASA and the planetary science community have been exploring the potential contribution(s) of stratospheric balloons to decadal class planetary science. Previous work $[1,2]$ has shown that significant progress can be made by answering over 20 percent of the key questions called out in the current Planetary Science Decadal Survey.[3] A recent study conducted on this subject revealed that a one meter aperture telescope, coupled with a pointing system that could achieve one arcsecond or better pointing stability, and an imaging instrument suite covering the near ultraviolet (NUV) to mid infra-red MIR) spectrum, namely $300 \mathrm{~nm}$ to 5 micron wavelengths, would be able to make significant contributes to planetary science. Such a platform, if designed correctly, has the potential of providing new and high-value science even after repeated flights. This is possible because of the 
breadth of available targets, the science questions addressable, and the variety of observing opportunities. The recent development and system demonstration afforded by the BOPPS mission underscores the potential balloon-borne payloads have for planetary science studies. The BOPPS predecessor mission, BRRISON, which stands for Balloon Rapid Response for ISON) showed that balloon payloads can be a rapid response platform to time critical science opportunity such as was the sun grazing comet, ISON. While BRRISON demonstrated the rapid response capability, it was not able to make scientific observations due to an anomaly early in the flight. Because of a new set of high value science targets, the favorable landing experienced by the BRRISON hardware, and the need to characterize the viewing and demonstrate the instruments and platform, it was decided to refly the hardware in the 2015 Fort Sumner campaign. This paper provides a brief summary of the science and engineering contributions of BRRISON and BOPPS.

BOPPS, for the first time on a balloon flight, demonstrated the imaging improvements realized by a fine-steering mirror incorporated in the visible optical system. The contributions of the BRRISON and BOPPS development and flights are discussed in Section 3 as is a discussion of the instruments used.

A recent report [2] discussed several features of a potential balloon borne platform that, if realized, would enable even more missions and science studies. In addition to larger apertures and better pointing, other desired capabilities are modular design concepts and systems to reduce risk of damage during landings.

The mission level enhancements being developed by NASA's Balloon Program Office is expected to contribute to new science studies. Longer flights with Ultra-Long Duration Balloons (ULDB's) as well as the planned launches from more mid latitude sites are two planned capabilities [4] that will offer additional science opportunities.

\section{Summary of Potential Contributions of Balloon Borne Payloads to Planetary SCIENCE}

\section{Potential Advantages of Balloon-based Science Platforms}

There are several key factors that allow balloon borne telescopes to make significant scientific contributions and/or support the planetary science community. Some of these include: 1) the space-like viewing environment at the typical $30-35 \mathrm{~km}$ float altitudes,[5] 2) the potential ability for long duration observations, or a regular cadence/frequent observations of targets, 3) low cost compared to space and airborne missions, 4) potential for rapid response, 5) the ability to repeat missions, 6) ability to easily upgrade instruments and gondola systems, and 7) short-mission cycles allowing for a large number of scientists to participate and get timely science data. Balloon-borne platforms are an excellent platform (especially for early career scientists, engineers, and project managers) to quickly build much needed experience, and get science results. Due to the broad set of expected science questions balloon observations can support and the multitude of targets available, the platform would provide the highest science per dollar return if it were flown multiple times from various locations. This implies that perhaps the best approach is to treat this planetary science platform as robust, reusable, and flexible mission concept rather than a one time "disposable" gondola philosophy often used for other balloon based missions. This unique observatory approach has significant ramifications for the gondola and payload design development and operations, but ultimately the goal is more science, at lower cost and faster return.

\section{Potential Science Summary}

Table 1 provides a summary of the planetary science important questions, as described in the 2013 Planetary Decadal Survey,[3] that a balloon-borne telescope could achieve or make significant progress toward. This table is based on a study currently being implemented that considered the "Important Questions" found in the survey report. [2]

Table 1. Potential science contribution traceability to decadal survey "Important Questions" [ref].

\begin{tabular}{|l|c|c|c|}
\hline \multicolumn{1}{|c|}{ Category } & $\begin{array}{c}\text { Total \# of DS } \\
\text { "Important } \\
\text { Questions" }\end{array}$ & $\begin{array}{c}\text { \# Answered or } \\
\text { significantly } \\
\text { addressed }\end{array}$ & $\begin{array}{c}\% \\
\text { Addressed }\end{array}$ \\
\hline Small Bodies & 23 & 10 & $43 \%$ \\
\hline Inner Planets & 39 & 11 & $28 \%$ \\
\hline Major Planets & 39 & 6 & $15 \%$ \\
\hline Icy Satellites & 75 & 12 & $16 \%$ \\
\hline Mars & 48 & 3 & $6 \%$ \\
\hline Total & 194 & 42 & $21 \%$ \\
\hline
\end{tabular}

The data in Table 1 is based on a number of assumptions on gondola performance, telescope aperture size, instrument capabilities, and so on. None of the assumptions are considered extraordinary, and in fact, much of the science can be done with a system put together from subsystems that have already flown on prior missions. For example, telescopes with a $1 \mathrm{~m}$ or larger aperture have already flown on stratospheric balloons. Several missions such as Sunrise, STO and BOPPS have demonstrated better than 1" pointing stability. Figure 1 shows how many of the 42 questions referred to in Table 1 can be addressed, at least to some level, with a gondola using a $1 \mathrm{~m}$ aperture telescope, and pointing at the 1 "level. 


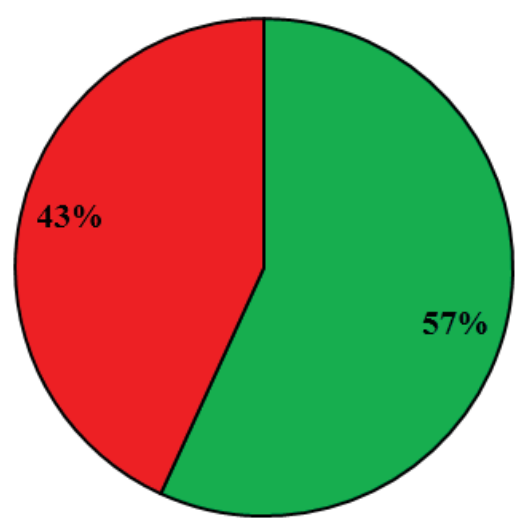

Figure 1 - Percent of Total Balloon-based Planetary Science Achievable with a $1 \mathrm{~m}$ Aperture and 1 arcsec Pointing Capability. [2]

There is clearly significant science that can be achieved with today's proven technologies and capabilities.

At a high level, top science measurements for such a platform are imaging applications and infrared spectroscopy. Due to the float altitudes $\left(\sim 120,000^{\prime}\right)$, diffraction limited observations are expected in the visible region with essentially no wave-front errors, and thus can be diffractionlimited. The altitudes also place the telescope above over 99.5 percent of the atmosphere, thus observations are improved at all wavelengths over ground and aircraft assets due to nearly ideal transmission in the mid and thermal infrared and significantly lower downwelling radiance. This combination enables a fundamental new capability over previous earth based observations with measurements in the mid-IR fundamental water and $\mathrm{CO}_{2}$ bands.

A number of specific science concepts were identified during a workshop held at NASA GRC on this topic and readers are referred to the website at http://microgravity.grc.nasa.gov/SSPO/SP/Balloon_Platfor $\underline{\mathrm{m} /}$ for a summary of results and a full list of the initial concepts conceived. An example of a science concept taking advantage of the imaging capabilities discussed is Venus observations to study atmospheric dynamics. Observations of giant planets and other bodies are also good candidates in cases when looking at whole discs or where high spatial resolution is not as critical.

Another important set of science contributions can be made with spectroscopy and addressing composition questions either identifying the existence of species or looking at abundances. A host of airless bodies are prime candidates for this type of science as is Mercury, the Moon, asteroids, icy satellites, and the hugely diverse complement of bodies in the outer solar system. Many concepts revolve around measuring secular variations that are almost uniquely enabled by these platforms over a range of timescales of seconds, to hours, days, months, and even years.

Summaries of potential science objectives have been covered in previous work, and in the remainder of this paper we focus on contributions made with the development of the
BRRISON and BOPPS missions flown on September $28^{\text {th }}$ 2013 and September $25^{\text {th }} 2014$ respectively, both from Fort Sumner, NM.

\section{CONTRIBUTIONS OF THE BRRISON AND BOPPS DEVELOPMENTS AND FLIGHTS}

\section{Contributions of BRRISON}

One of the notable contributions of the BRRISON development was demonstration that balloon based platforms can be a tool in the planetary science arsenal for quick response compared to space missions. As interest and attention grows for finding and categorizing Near Earth Objects (NEO's) [6] and as the science and amateur astronomy communities continue to find exciting new bodies, the need for rapid and repeated deployment of observing capability will increase. Other potential opportunities could be the leveraging of naturally occurring impacts (e.g., Jupiter and comet Shoemaker Levy) for unique opportunities to make compositional and dynamic observations.

In the case of BRRISON, the time critical target was Comet ISON discovered in September, 2012 and which died on November $28^{\text {th }}, 2013$ upon entering perihelion. This comet, believed to be on its first and perhaps only pass through the solar system from the Oort cloud, offered a unique chance to make abundance measurements of $\mathrm{CO}_{2}$ and $\mathrm{H}_{2} \mathrm{O}$. Until now, our ability to study this comet would have been exclusively left to observations based on instruments and space assets which have serendipitous instruments and capability to make the desired measurements. Ground assets are generally good candidates for quick response situations, but observations in certain critical bands may not be possible and atmospheric effects may make imaging challenging.

BRRISON demonstrated that balloon platforms have the potential for being a quick response tool. In the case of BRRISON, the mission was enabled by the fact that an existing telescope, with an aperture of $.8 \mathrm{~m}$, was available for immediate refurbishing and the basic avionics and control systems of a gondola were available to reuse. However, all other aspects of the gondola, including the structure, and most importantly the instrument systems were designed, built and tested specifically for the Comet ISON observing mission. While it is not recommended to rely on serendipitous existing hardware for future quick response opportunities, the BRRISON mission did indeed demonstrate the potential.

The better path toward quick response would be to have, under the NASA Planetary Science Division control, a gondola with flight proven avionics, a highly capable telescope with fine pointing system and a small team experienced with that hardware, available for near term redeployment. A set of instruments with relatively broad application, like what was defined in the study, [2] may also prove invaluable. Given these basic building blocks a response on the order of months may be realized. 
It is not envisioned that this hardware and team would be standing by waiting for an opportunity to arise. Rather the approach is to have planned flights perhaps on annual basis to accomplish competitively selected science goals. However, the same basic hardware and design, and a gondola designed to survive the landings would enable a redeployment to another target on very short order. The ability to retrieve a balloon payload is therefore another important feature for balloon based missions and something not available with space missions. So when a unique or high value opportunity arises, the science objectives can be redefined and the appropriate instruments can be installed or modified if needed for the new target. Figure 2 shows the BRRISON gondola which was built and launched in less than 9 months by a team from NASA GRC, Johns Hopkins Applied Physics Laboratory (APL) and the Southwest Research Institute (SwRI).

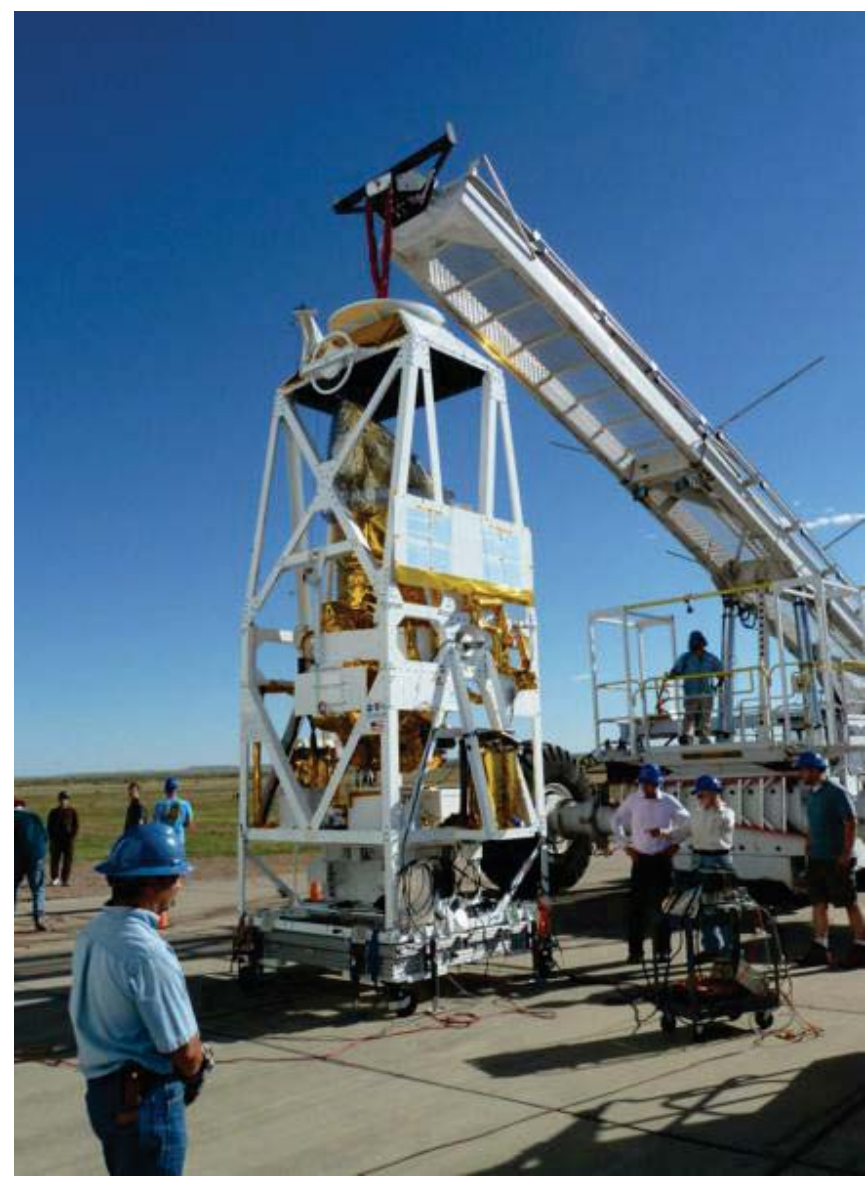

Figure 2 - BRRISON Gondola during pre-launch check.

Another important contribution of the BRRISON development effort was the procurement and fabrication of most of the required instruments and optical benches that can serve as a basic instrument suite for future flights. The BRRISON team designed and fabricated a gondola that could hold a 1-m telescope and protect it under normal landing scenarios. Additionally, the development included two optical benches complete with a NUV-Vis and NIR-MIR cameras and filter wheel assemblies. The cameras and supporting systems allow observations from $300 \mathrm{~nm}$ to about $5 \mu \mathrm{m}$. As can be seen in Figure 3, this captures a significant part of the observations called out in the decadal that a balloon borne telescope is suitable for.

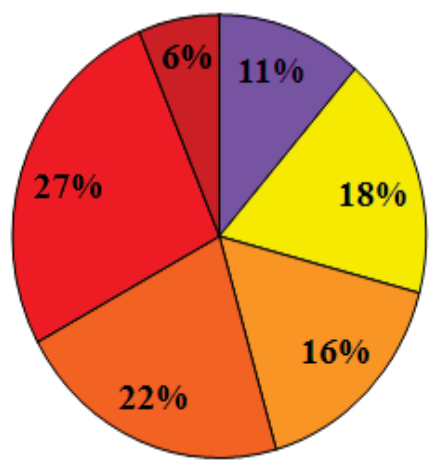

$\square$ NUV (300 - 400nm)

$\square$ Visible (400-750nm)

$\square \operatorname{NIR}(750 \mathrm{~nm}-1 \mu \mathrm{m})$

$\square \operatorname{SWIR}(1-2.5 \mu \mathrm{m})$

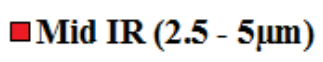

$\square$ Thermal IR (5-12+ $\mu \mathrm{m})$

Figure 3 -Science capture by observing spectrum.

A diagram of the NUV-Vis optics bench, the "UVVis" camera and the fine steering guidance camera used for the BRRISON mission is shown in Figure 4.
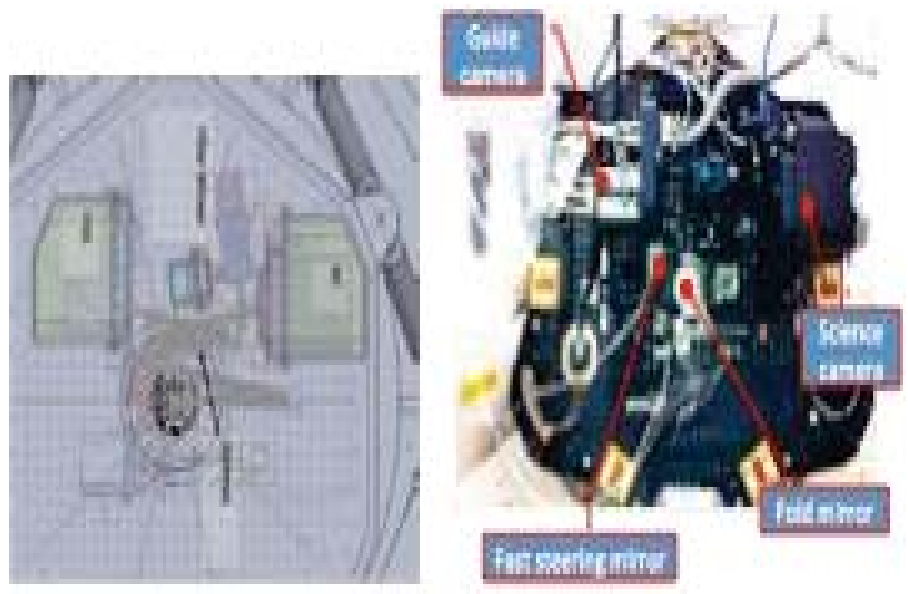

Figure 4 - NUV-Vis optical diagram.

A number of significant contributions were made during the pre-launch ground tests and during the ISON (September 2013) flight. Unfortunately the comet ISON was not observed during the flight due to an anomaly that locked the telescope in an unfavorable position before the comet became visible. Hang tests from the ground showed that the gondola's coarse pointing system could keep a target within a 20 " field of view, even in the presence of wind gusts, and further, that the guide camera and FSM could reduce image motion. Uncompensated hang-test images had point spread functions (PSFs) with 3" widths (full-width at half maximum, FWHM), but long exposures with the FSM compensating for pointing errors had PSFs of 1.5", which is close to the theoretical improvement of a PSF when tip-tilt errors are removed. 
One of the objectives of the BRRISON (and also BOPPS) flight was to demonstrate the capability and use of a fine steering mirror system to improve visible imaging of extended targets. This capability will significantly enhance the efficacy of the system for planetary science especially for addressing science goals needing high spatial resolution. A second goal was to demonstrate arecsec level pointing of the gondola and telescope control system to enable the FSM to operate effectively and to provide sufficient stability to make IR spectral measurements without the use of an FSM. The ground testing of this entire system was conducted in conditions that are significantly more severe than what is seen during flights. The gondola pointing control system utilizes a guidance camera to accurately determine pointing errors and then relies on a one or two stage control system to maintain the required pointing precision. The first control stage uses a reaction based system with spinning masses to maintain arcsecond-level azimuth and elevation pointing when at float. Even during strong wind gusts the system on BRRISON ground tests was able to maintain a target to within about 1015 " and under calm conditions maintained better than arcsecond stability. The second stage, embedded into the visible channel only, used a fine guidance camera to actively control a mirror assembly that quickly compensated for motion not taken out by the gondola and telescope pointing control.

\section{Contributions of the BOPPS mission}

Due to new high-value science targets and the anomaly with the BRRISON flight, which prevented characterization of the instruments, the decision was made to refly the BRRISON hardware correcting the anomaly causes under a new name, BOPPS, because Comet ISON no longer existed. The development effort for BOPPS included a redesign of the telescope stow latch, replacement of the "old" commercial computers with ruggedized new ones capable of operating without a pressure vessel, improved pointing control software, better calibration science instrumentation, and increased system level testing which was simply not possible on BRRISON due to Comet ISON schedule pressures.

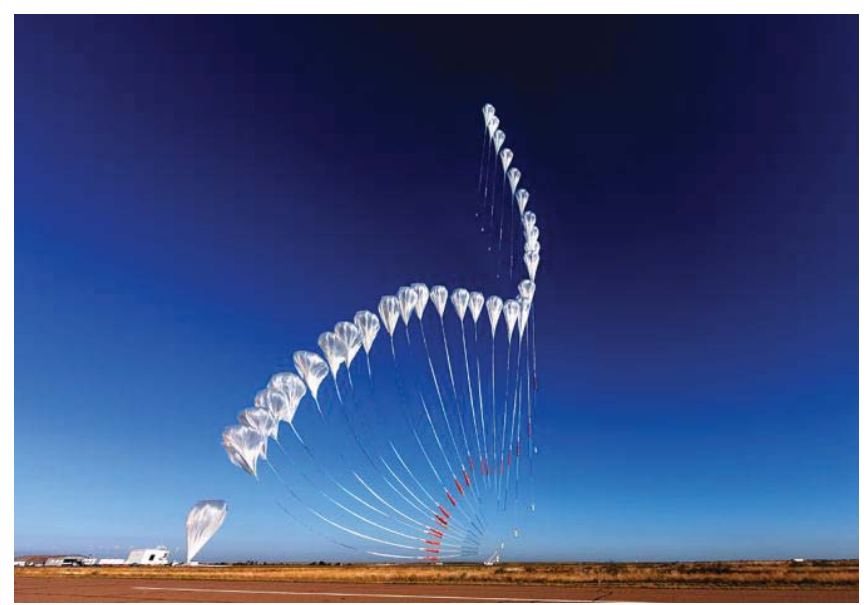

Figure 5 - Time lapse images of BOPPS launch. (Geoff Brown, JHU-APL)
BOPPS was launched from Fort Sumner on September $25^{\text {th }}$, just one day after Comet Siding Spring, considered the prime science target for this mission, came into a viewable altitude above the horizon. Figure 5 shows an interesting perspective of the launch with time lapse mosaic of still images. Along with Comet Siding Spring a number of other targets had been identified and a notional observation timeline was developed. Other observation targets for this date in the launch window included comet PanSTARRS, Comet Jacques, the large asteroids Ceres and Vesta, the planet Uranus, and a number of stars/calibration targets.

Weather conditions delayed launch of BOPPS by approximately 2.5 hours which pushed gondola 'commissioning' at float to overlap with the planned Vesta observations so that they would no longer be made. Commissioning the gondola and instruments ensured that all instruments, the telescope and the various sub-systems were functional and responding as expected and to 'calibrate' the gondola pointing control system at the temperatures encountered during float. It may be that the sky background during the day was significantly brighter than encountered during flights in Antarctica using these same star cameras. The determination of telescope attitude during daylight hours was slower and ultimately less accurate than predicted. Also, the alignment between the star camera and telescope was different at float than on the ground, as expected again due to operation at a colder temperature at float. Together, though these environmental and alignment differences slowed target acquisition and ability to track during daylight hours. These are tractable issues that can be addressed before any next flight by this or another platform.

The general observing approach was to provide the instrument teams the necessary time to achieve their requirements with the observing windows afforded by the target and within the system constraints. Recall that BOPPS has both a Ultra-Violet/Visible optics bench and instrument (UVVis) (Figure 4) as well as an Infra-red imager (BIRC) (Figure 6) with its own optics bench. The system is designed so that one or the other instrument can operate at a time but not simultaneously. This meant that the observing time needed to be shared by the instruments thereby constraining observation flexibility. Despite these time challenges the science, operations, and engineering teams met all the flights' Level 1 requirements. 


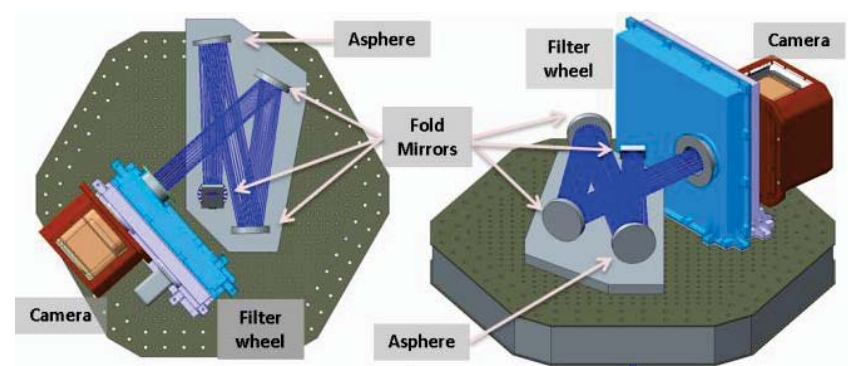

Figure 6 - Two views of the IR optical bench. (Light enters at the center of the hexagonal bench, after bypassing the UVVis optical bench, and is bent into the plane of the bench at the first fold mirror. Light is then collimated at the asphere, and after two more fold mirrors, it passes through a $\mathrm{CaF}_{2}$ window, through the nine-position filter wheel, and into the IR camera.)

The first science target observed by BOPPS was the Oort Cloud comet Siding Spring, after which the dwarf planet Ceres was observed and then a second Oort Cloud comet, Jacques, all using the multispectral BIRC instrument. The BIRC instrument is described in detail in Reference [7]. Table 2 summarizes the BIRC observations, showing the sun distance $r_{H}$ of the target, the Earth distance $\delta$, the solar phase angle, the calibration star employed, and successful detections. $\mathrm{R}$ band is in the visible at band center $641 \mathrm{~nm}$. The $2.7 \mu \mathrm{m}$ band is for water, and the $4.27 \mu \mathrm{m}$ band is for $\mathrm{CO}_{2}$. All three targets were detected at $2.7 \mu \mathrm{m}$ and Jacques was also detected at $4.27 \mu \mathrm{m}$.

One of the goals of the BOPPS missions was to characterize how well the gondola pointing system performed in flight. Figure 7 shows that gondola pointing was well below 1 arcsec, the desired target for pointing stability.

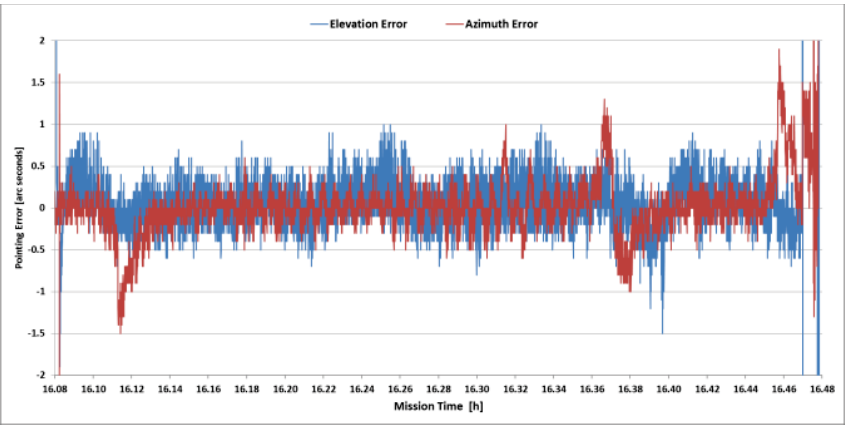

Figure 7 - Gondola Pointing Stability at Float Altitude

The UVVIS team was next given time to demonstrate functionality and characterize performance of the fine steering mirror (FSM) system at float conditions. The team selected a target star, Polaris in this case, and explored FSM performance including with and without running the IR system mechanical coolers, as they caused vibrations.

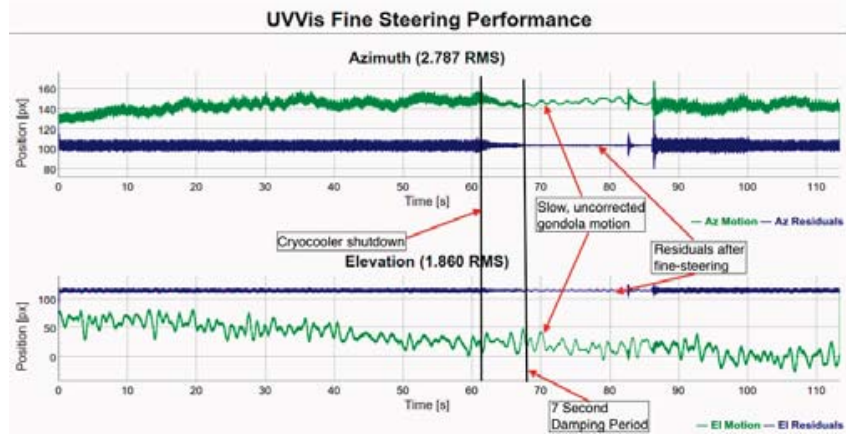

Figure 8 - Guide star positions in azimuth (upper plot) and elevation, both uncorrected (green) and corrected with the FSM (blue). The left-hand side of the plot shows the FSM performance when the cryo-cooler is $\mathrm{ON}$. The cooler was turned off at $t=62 \mathrm{~s}$, followed by a $7 \mathrm{~s}$ damping time.

In the September 2014 BOPPS flight, the UVVis bench was able to take a few image sequences of Polaris during the daytime segment of the flight. The image sequences spanned windows in which the cryo-cooler was turned off for ten or twenty seconds. Figure 8 shows the correction in elevation (EL) and azimuth (AZ) as the cryo-cooler was turned off. The rms pointing errors were 280 mas and 165 mas in AZ and EL, respectively, when the cryo-cooler was $\mathrm{ON}$. The rms pointing errors improved to 33.3 mas and 58.1 mas in AZ and EL when the cryo-cooler was OFF. The overall stability was 66 mas with the cryo-cooler off, surpassing the BOPPS Level-I goal of demonstrating sub-0.1" pointing stability. Figure 8 reflects the improvements of the FSM system affords the UVVis images. It should be noted that this test utilized a position correction control law with a low pass filter at $.5 \mathrm{~Hz}$. If a less conservative level of $3 \mathrm{~Hz}$ were used the performance is expected to be even better. The need to maintain within $\mathrm{BIRC}$ detector specified operating temperatures required that the cooler be turned back on shortly after demonstrating FSM performance at these parameters.

The most desired science observation of the BOPPS flight was IR imaging of Comet Siding Spring. Comet Siding Spring was on a trajectory to pass within 140,000 km of Mars on October $19^{\text {th }}, 2014$ and a host of NASA assets were engaged in cooperative observations of the Comet. The instruments and the advantageous viewing location allowed BOPPS to offer unique data and science contributions to this observing campaign. Figure 9 are images of Polaris with the same instrument but one taken during ground testing while the other is during float. The improvement in background signal is clear as is the $2+$ orders of magnitude improvement in signal intensity reaching the instrument. Figure 9 is a visual example of the significant advantage a balloon-borne observatory offers in terms of viewing quality over ground based assets. 


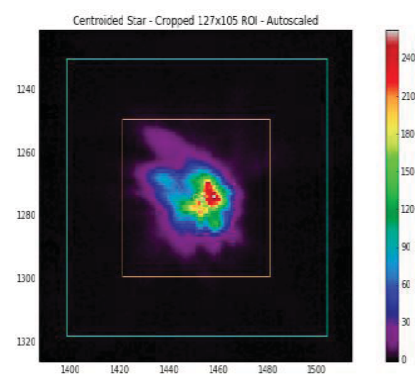

Figure 2: Float image of Polaris, scaled by $\sigma$ above sky background

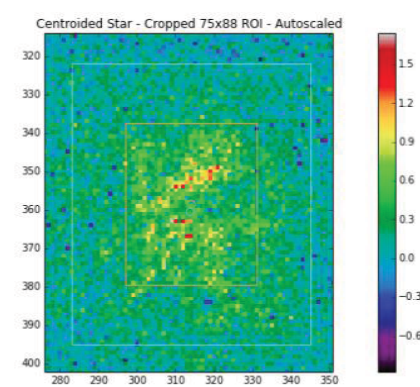

Figure 3: Ground image of Polaris, scaled by $\sigma$ above sky background
Figure 9 - Polaris Imaging with the UVVis Instrument from the Ground and at Float Altitude

After commissioning, capturing and focusing star images, observing Ceres and demonstrating the UVVis and FSM system, the BOPPS team prepared for the short viewing window that opened up when Comet Siding Spring came above the horizon. The comet was expected to be approximately 9.6 magnitude brightness but uncertainty in the residual atmospheric affects and Comet brightness introduced some uncertainty. The team did manage to capture observations of the faint comet as shown in Figure 10. Figure 10 is an image taken by BIRC of Siding Spring through the 2.7 micron narrow-pass filter. The comet is clearly observable in this water emission band.

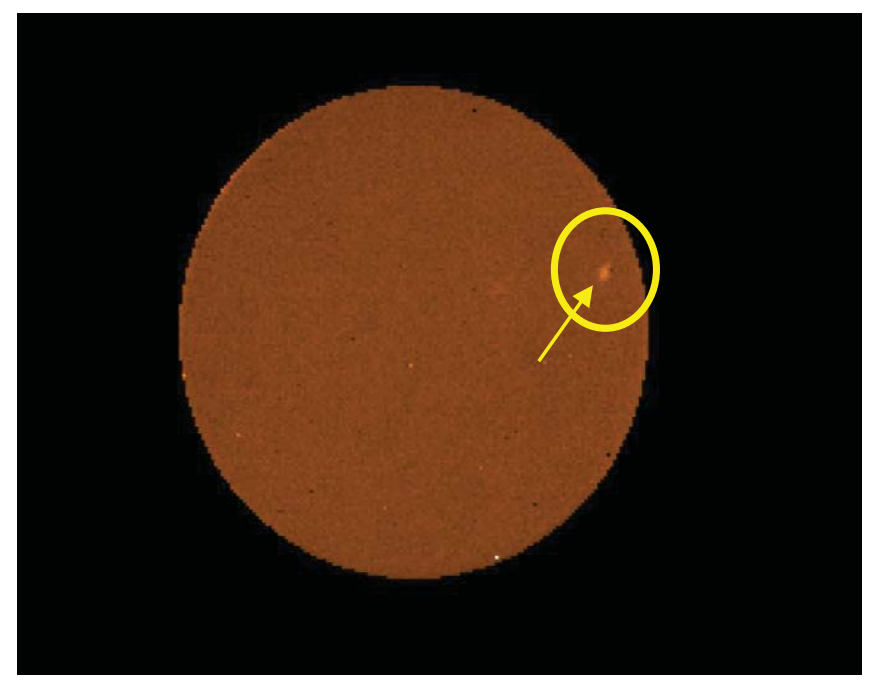

Figure 10 - BIRC image with Comet Siding Spring in Field of View (FOV) with $2.7 \mu \mathrm{m}$ filter (background not subtracted)

While BOPPS was not able to observe PanSTARRS because commissioning delays and attitude determination issues during the daytime, another Oort cloud comet, Jacques, was located by the science team and imaged with the BIRC instrument in the early evening.

Figure 11 is an image of Comet Jacques with BIRC using the $4.27 \mu \mathrm{m}$ filter.

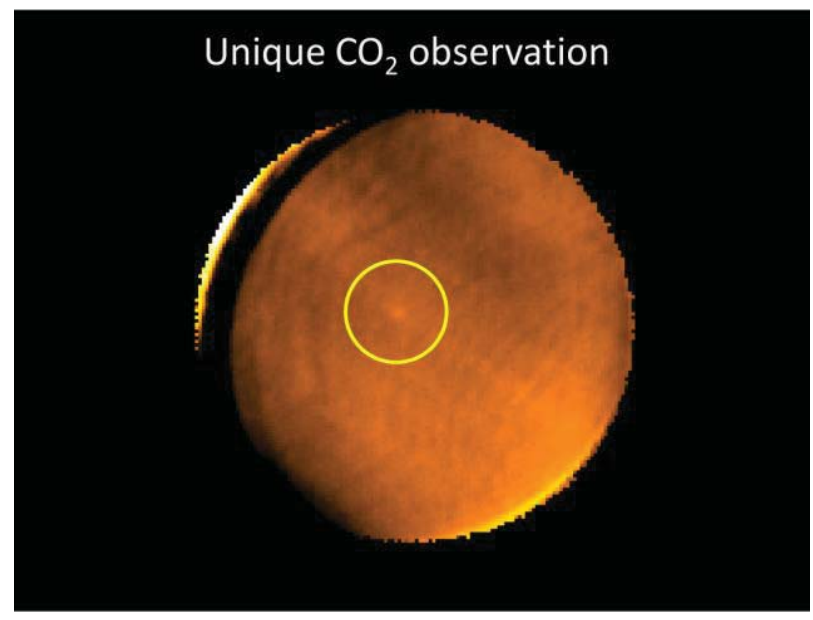

Figure 11 - BIRC Image of Comet Jacques at $4.27 \mu \mathrm{m}$.

Further night time imaging was planned for the UVVis instrument but nighttime focus difficulties and the termination of the flight due to overflight of a populated area precluded further UVVIS images. The flight was terminated west of Amarillo at approximately 3:30am.

The BOPPS payload survived the landing in good condition despite the $11+\mathrm{g}$ impact at landing. Figure 12 shows the BOPPS gondola as it landed after the flight.

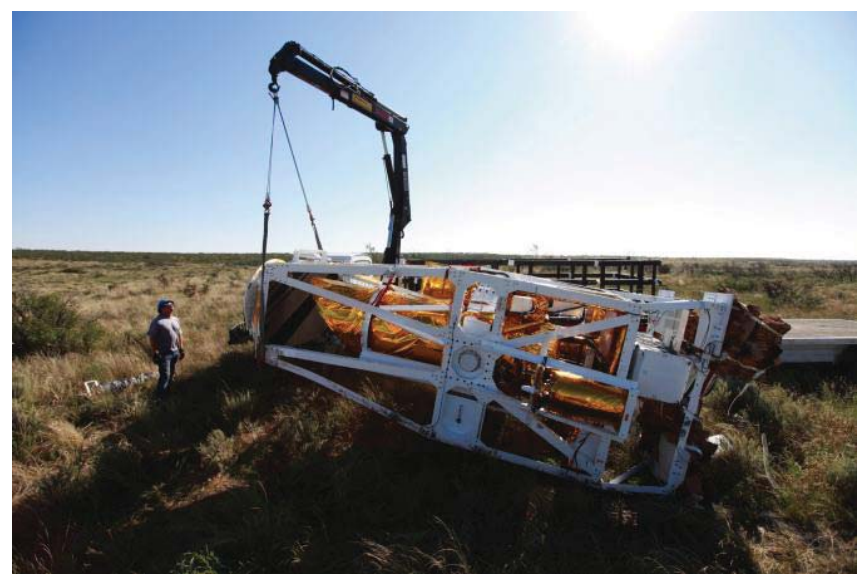

Figure 12 - BOPPS gondola - post flight. . It landed vertically and came to rest on its side.

The BOPPS telescope is planned for use on another mission over Antarctica in the winter of 2016

\section{Potential Future System and Enhancements To EnAble More Planetary SCIENCE}

As capable as the BOPPS payload is, it will not be able to achieve the breadth of the planetary science that could be realized from stratospheric balloons. As discussed earlier in this paper and in previous work,[2] a baseline system would require a telescope with at least a $1 \mathrm{~m}$ aperture, a hyperspectral capability in the IR, and a longer mission duration. The BRRISON/BOPPS telescope is $0.8 \mathrm{~m}$ in diameter. The 
telescope and gondola system would need to be light weighted in order to be flown with ultra-long duration balloons, a potential enabler for some planetary science. The BRRISON/BOPPS gondola was not designed to that requirement. For these and similar reasons, a new telescope and gondola will need to be designed and built to achieve the breadth of science that the platform can support.

A project is being formulated at NASA GRC to explore the desired features of a balloon-borne gondola, develop the detailed requirements, and possibly begin implementation to accomplish the goal of addressing the host of science achievable from stratospheric balloons. This effort is currently known as the Gondola for High Altitude Planetary Science (GHAPS) project. The project will leverage the BRRISON and BOPPS missions and hardware to the extent possible, NASA's Balloon Program Office (BPO) expertise, as well as those of others in government and industry to make this capability available to planetary scientists as soon as possible. This project is still in pre-formulation phase, but some aspects of the ongoing study are available. For example, a concept gondola has been generated in the study.[2] The concept utilized the BPO developed Wallops Arc-Second Pointer (WASP) as the pointing system. While WASP was assumed for the concept, there are a number of pointing systems that may be available, including the ones used on BRRISON and BOPPS, as candidates for a GHAPS pointing system, and therefore, an assessment will be conducted by the GHAPS project to determine which pointing system is most suitable for the objectives of the project. Figure 13 depicts a concept (telescope casing and some other hardware removed for clarity) developed under the study.

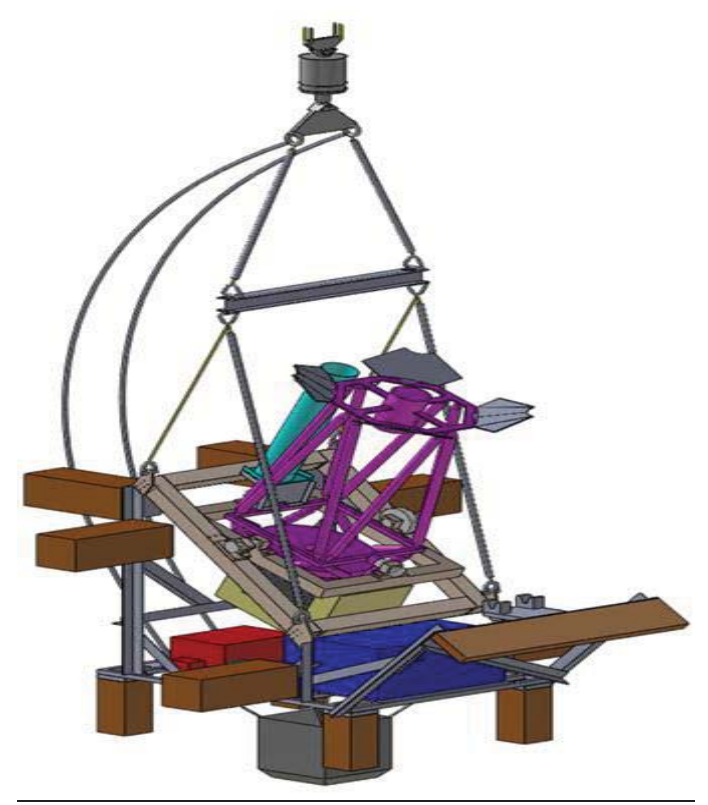

Figure 13 - Preliminary gondola concept.

In addition to developing requirements for a baseline gondola, the GHAPS project will also assess known needs (and offer design solutions) as the platform evolves to tackle new and more challenging goals. For example, to enable extended observations, especially those requiring day and night views, long flights in mid latitudes will be required. This will necessitate over sea flight with the possibility that the gondola may suffer an ocean landing. The payload will need to be appropriately protected for that scenario.

The GHAPS flight platform will need to be capable of being readily disassembled at its landing site to enable transportation by the recovery vehicles without causing further damage to the gondola, and re-used for at least several missions. Initial trade assessments have been completed on the GHAPS primary structure which will lead to a lightweight flight platform capable of being quickly disassembled, perhaps without the use of tools, in the frigid Antarctic climate. Limiting human exposure time in the Antarctic while being able to break down the GHAPS flight platform and its instruments into manageable packages for loading into recovery aircraft will be a goals for GHAPS development. The challenge will be exacerbated by the requirements for a light-weight structure, and a structure capable of being re-used.

In addition, the vastly different at-altitude thermal environments of different BPO launch sites create unique engineering problems. Antarctic missions experience daylight for the entire mission and see a relatively warm temperature which presents a challenge of keeping temperature sensitive instruments (ex. infrared) cold. In contrast, a mid-latitude flight will see constant day-night cycles that result in stresses on all of the subsystems as both day and night observations are desirable. Power required to protect critical systems after landing, particularly in the harsh Antarctic environment will be an additional driver for the thermal design of the GHAPS flight platform and the power system.

Several other trade assessments have been completed including power management, power generation and storage, power distribution, software, and avionics. All of these assessments will be revisited as top level requirements are finalized, but do serve as further proof of concept for a longduration balloon-borne observatory.

Initially, the GHAPS flight platform will be designed to protect itself and its science instruments against a worst-case landing on terra firma. The GHAPS team will be assessing various landing systems to protect the flight platform, including crushable pads and deflating air bags. In later incarnations, GHAPS will also provide protection against landing in water and still meet its mission re-use capability. Air bags could provide floatation at sea as well as a cushion for a land impact. Locating beacons and water-tight enclosures may be another challenge that the GHAPS team will need to consider.

There are several other assessments that will be considered as the GHAPS is formulated with the intention that the platform can be enhanced gracefully over time as it accomplishes new and exciting science in tougher operating and landing environments. It is the intent of the GHAPS project that the 
science performed by the platform would be competed via an announcement of opportunity. The exact vehicle for this is still being determined.

It should be noted that, as of paper submission, no decisions have been made to fully implement GHAPS or how that implementation may occur.

\section{SUMMARY}

Prior work $[1,2,4]$ has shown that stratospheric balloon borne gondolas can offer planetary scientists a highly capable platform for exciting new science observations. The platform enables observations in critical bands that are not feasible from the ground or even airplanes. With a balloon borne observatory, this science can be achieved in a very cost effective manner. The platform can offer a capability for extended observations dedicated to planetary science, something not possible from Earth today. Another key attribute, as demonstrated by the BRRISON development, is the quick response capability of this platform.

The BRRISON development was in response to the discovery of comet ISON and the decision to try and make unique observations of the comet as it approached the sun. BRRISON was designed and built in less than nine months and launched from Fort Sumner on September 28 ${ }^{\text {th }}, 2013$ leveraging hardware and experience from the APL and SwRI team, and the Solar Terahertz Observatory (STO) mission [8]. The BOPPS mission addressed the anomalies experienced by BRRISON and made unique science measurements of Oort cloud comets and other targets in its one day flight. It also demonstrated the improved viewing conditions in the visible and the improvements possible with a FSM system. The BRRISON/BOPPS missions show that a balloon-borne observatory would be a significant contribution to the planetary science arsenal.

NASA GRC has begun the GHAPS project, the goal of which is to develop detailed requirements and begin developing a gondola that can achieve the host of planetary science addressable from stratospheric balloons. The GHAPS gondola will be designed to fly on ultra-long duration balloons, incorporate a minimum 1-m aperture telescope, and a pointing system capable of controlling the gondola to 1 " or better. The BOPPS proven instruments (NUV-Vis through mid- Infrared) are expected to form the basis of the core instrument package available for scientists to use in their observations on first generation flights by the GHAPS gondola. The development effort will consider options for future needs such as more capable instruments, surviving ocean landings and more demanding ground landings, and modular and flexible design and structure to name a few.

\section{REFERENCES}

[1] Kremic, T., Hibbitts, K., Young, E., Landis, R., Noll, K., and Baines, K., "Assessing the Potential of Stratospheric Balloons for Planetary Science," IEEEAC Paper \#2074, 2013 IEEE Aerospace Conference, Big Sky, MT, March 29,2013

[2] Dankanich, J. "Planetary Balloon-Based Science Platform Evaluation and Program Implementation, Final Report," NASA Technical Report, unpublished

[3] "Vision and Voyages for Planetary Science in the Decade 2013-2022," The National Academies Press, URL: http://www.nap/edu, 2011

[4] http://spaceflightsystems.grc.nasa.gov/SSPO/SP/Balloon Platform/agenda.html

[5] "Space quality data from balloon-borne telescopes: The High Altitude Lensing Observatory (HALO)," Astroparticle Physics, Vol. 38, pgs. 31-40, 10/2012.

[6] Sec. 321 George E. Brown, Jr. Near-Earth Object Survey Act, Public Law 109-155-Dec. 30, 2005, http://www.gpo.gov/fdsys/pkg/PLAW109publ155/pdf/PLAW-109pub1155.pdf

[7] McMichael, R. T., et al, "BRRISON IR Camera (BIRC)," Proc. SPIE. 9145, Ground-based and Airborne Telescopes V, 91452W. (July 22, 2014) doi: 10.1117/12.2057619

[8] Walker, C, et al, "The Stratospheric THz Observatory (STO), Proc. SPIE. 7733, Ground-based and Airborne Telescopes III, 77330N (July 28. 2010); doi: $10.1117 / 12.857765$

\section{BIOGRAPHIES}

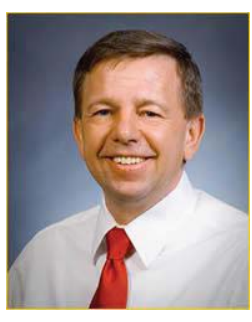

Tibor Kremic is currently working at NASA Glenn Research Center in the Science Project Office where he guides science related initiatives at the GRC. Formally he served on a detail as the assistant Division Director for Planetary Science where he contributed to the management of NASA planetary science program. Prior roles included management of organizations and project or program manager on a variety of NASA efforts, including NASA's In-Space Propulsion Technology Project. He also serves on a number of panels and teams focusing on science or space related technologies. Among the other duties he was the lead for the study discussed in this paper. 


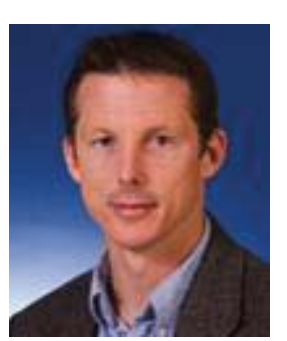

Charles A. Hibbitts received a B.A. in Physics from Cornell University in 1989. After serving in the military, he earned a degree in Geology from the University of New Mexico, and an MSc and PhD. in Geology and Geophysics at the University of Hawaii in 2001. His dissertation topic was understanding the surface compositions of the icy satellites of Jupiter by analyzing data returned by the Near Infrared Mapping Spectrometer aboard the Galileo spacecraft. Since then, Dr. Hibbitts has expanded his interests to other bodies of the solar system, including asteroids, the Moon, and the Earth.

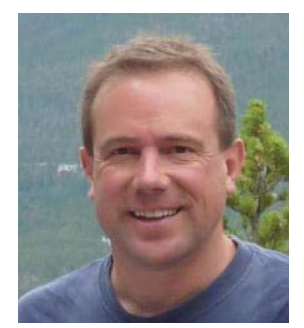

Eliot $\boldsymbol{F}$. Young received an $A . B$.in Physics from Amherst College in 1984, an S.M. in Aeronautical Engineering from M.I.T. in 1987, an S.M. in Earth, Atmospheric and Planetary Science (EAPS) from M.I.T. in 1990, and an Sc.D. from M.I.T. (EAPS) in 1992. He is currently a Principal Scientist at Southwest Research Institute in Boulder, CO, in the Department of Space Studies. His current areas of study include the surfaces and atmospheres of Pluto, Triton, Eris and other large Trans-Neptunian Objects, as well as the distributions of aerosols and trace gases in Titan's atmosphere and the wind fields on Venus.

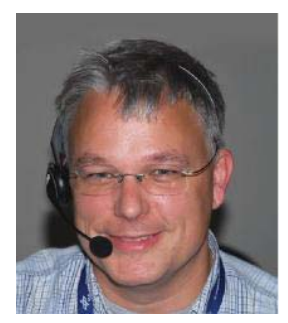

Rob R. Landis is currently a Program Officer within the Science Mission Directorate at NASA. Previously, Mr. Landis was the Technical Manager/Senior Engineer at the NASA Goddard Space Flight Center/Wallops Flight Facility. Before that he was the head of mission operations for the "robotic recon experiment" at the NASA Ames Research Center as well as part of the Lunar Surface Systems Project at the NASA Johnson Space Center. He previously worked at the Space Telescope Science Institute (STScI) on the Hubble Space Telescope, at the Goddard Space Flight Center on the Rossi X-ray Timing Explorer, and at the Jet Propulsion Laboratory on Cassini-Huygens and the Mars Exploration Rovers. Mr. Landis has a B.S. in astrophysics from Michigan State University and a M.S. in space studies from the University of North Dakota.

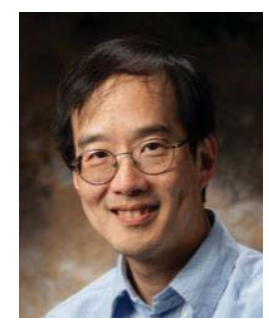

Andrew F. Cheng received an $A . B$.in Physics from Princeton University in 1971 and a Ph.D. in Physics from Columbia University in 1977. He is Chief Scientist for the Space Department at the Johns Hopkins Applied Physics laboratory. He was Deputy Chief Scientist for Space Science in NASA's Science Mission Directorate, at NASA Headquarters, from 2007 to 2008. He was a science team member for the Galileo,
Cassini, Messenger, and Hayabusa missions. He was Project Scientist for the Near Earth Asteroid Rendezvous (NEAR) mission. He is currently Principal Investigator for the Long Range Reconnaissance Imager (LORRI) instrument on the New Horizons mission to Pluto and the Kuiper Belt.

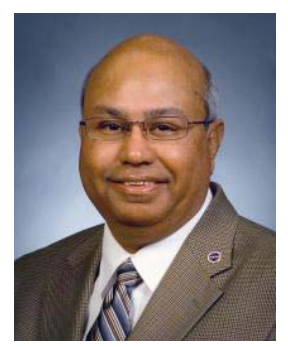

Rafat R. Ansari, Ph.D., is a Senior Scientist at NASA Glenn Research Center. His association with NASA started in 1988 conducting microgravity fluids physics experiments on-board space shuttle orbiters, Mir and International space station. He is BRRISON's and GHAPS's GRC Science Lead. He is well-known in the area of biomedical optics and experimental ophthalmology with over 100 scientific publications. Currently, he is serving on the Editorial Boards of the Journal of Biomedical Optics, and the Journal of Health Information Science and Systems. Among many honors, Ansari is a recipient of the Public Service Medal, two NASA's Space Act Awards, the Abe Silverstein Medal, STARS Award from the State of Texas, Service to America Medals finalist for Science and Environment, the NASA Exceptional Scientific Achievement Medal, and the Innovation Award from the US Department of Health and Human Services, the Pioneer in Medicine Award from the Society of Brain Mapping and Therapeutics, and honors from both the Ohio State Senate and the U.S. Senate.. He is a Fellow of the SPIE (The International Society for Optics and Photonics), an inventor (three patents) and a licensed pilot.

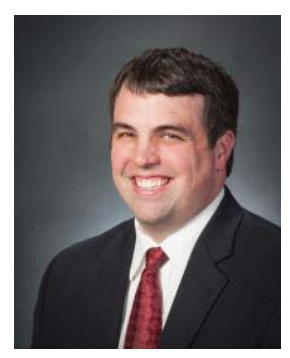

Matthew Dolloff is currently serving as the acting Project Manager for the Gondola for High Altitude Planetary Science (GHAPS) at the NASA Glenn Research Center. Prior to his work as the GHAPS Project Manager, Matthew served as the Product Lead Engineer for the Advanced Stirling Radioisotope Generator Control Unit. Matthew earned a BS in Electrical Engineering and a BS in Computer Science from the University of Wisconsin - Madison, and a $M S$ in Electrical Engineering from Cleveland State University. 